\title{
Aspectos clínicos e hematológicos em cães submetidos à fluidoterapia intravenosa, nutrição enteral e parenteral
}

[Clinical and hematological aspects in dogs alloted to intravenous fluid therapy, enteral and parenteral nutrition]

\author{
R.C. Valadares ${ }^{1}$, M.S. Palhares ${ }^{2 *}$, A.L.F. Bicalho ${ }^{3}$, C.R. Turchetto Jr. ${ }^{3}$, \\ M.D. Freitas ${ }^{3}$, J.M. Silva Filho ${ }^{2}$, A.U. Carvalho ${ }^{2}$ \\ ${ }^{1}$ Aluno de pós-graduação - EV-UFMG \\ ${ }^{2}$ Escola de Veterinária - UFMG \\ Caixa Postal 567 \\ 30123-970 - Belo Horizonte, MG \\ ${ }^{3}$ Médico veterinário autônomo
}

\begin{abstract}
RESUMO
Avaliaram-se peso vivo, temperatura retal, comportamento, escore fecal e hemograma em 20 cães, sem raça definida, distribuídos aleatoriamente em quatro grupos experimentais com cinco animais cada, duas fêmeas e três machos, submetidos ao arraçoamento padrão (grupo I - controle), à fluidoterapia intravenosa (grupo II), à nutrição enteral por gastrostomia (grupo III) e à nutrição parenteral total (grupo IV). Os tratamentos duraram sete dias, precedidos por dois dias de jejum alimentar. Os animais do grupo II apresentaram a maior perda de peso $(\mathrm{P}<0,05)$, e os do grupo IV flebite, já no segundo dia de tratamento. Não foram observadas alterações no eritrograma $(\mathrm{P}>0,05)$. Os animais do grupo II apresentaram valores abaixo dos de referência para hemácias, hemoglobina e hematócrito, sugerindo anemia normocítica normocrômica. A fixação da sonda gástrica, via endoscopia, levou a alterações no número total de leucócito $(\mathrm{P}<0,05)$, causando desvio à esquerda regenerativo nos animais do grupo II. A velocidade de administração da solução parenteral deve ser monitorada quando se utiliza o acesso venoso periférico.
\end{abstract}

Palavras-chave: cão, fluidoterapia, nutrição parenteral, nutrição enteral

\begin{abstract}
Body weight, rectal temperature, behavior, fecal score and hemogram were evaluated in 20 crossbred dogs, randomly alloted in four experimental groups, being five animals in each group, two females and three males. Those animals were alloted to standard feeding (group I - control), intravenous fluid therapy (group II), enteral nutrition through gastrotomy (group III) and total parenteral nutrition (group IV). The treatments lasted seven days, preceded by two days of fasting. The animals of group II showed the highest weigth loss $(P<0.05)$, and the animals of group IV showed phlebitis, since the second day of treatment. No changes were observed in red blood cell count $(P>0.05)$, however, the animals of group II showed lower values of erythrocytes, hemoglobin and hematocrit, suggesting normocytic normochromic anemia. The gastric tube placement, through endoscopy, led to alteration of the total number of leukocytes $(P<0.05)$, causing a regenerative left shift in the animals of group II. The administration rate of the parenteral nutrition solution must be monitored when a peripheral venous access is used.
\end{abstract}

Keywords: dog, fluid therapy, parenteral nutrition, enteral nutrition

Recebido em 12 de agosto de 2004

Aceito em 2 de janeiro de 2006

*Autor para correspondência (corresponding author)

E-mail: palhares@vet.ufmg.br 


\section{INTRODUÇÃO}

Nos últimos 25 anos, a comunidade médica desenvolveu sistemas práticos para a nutrição parenteral total, líquidos enterais para nutrição enteral superior e técnicas cirúrgicas de fácil colocação de tubos de alimentação enteral. Essa tecnologia, aplicada recentemente em pacientes veterinários, confirma o sucesso documentado na medicina humana (Donoghue, 1992).

O suporte nutricional terapêutico visa fornecer, via enteral ou parenteral, os nutrientes necessários para manutenção e recuperação do paciente hospitalizado. Uma vez que a inapetência e inanição são situações comuns em animais enfermos, levando, muitas vezes, a quadros severos de desnutrição e agravamento da enfermidade, o suporte nutricional é de grande interesse clínico. Um estado nutricional adequado otimiza a resposta a tratamentos clínico-cirúrgicos, impede a diminuição da resposta imune, minimiza a perda de massa corporal, favorece a cicatrização de feridas e, conseqüentemente, diminui o tempo de permanência do animal em um ambiente hospitalar hostil (Armstrong e Lippert, 1988; Simpson e Elwood, 1994). A perda de $10 \%$ do peso corporal e/ou períodos de anorexia superiores a quatro dias são indicativos de uso do suporte nutricional adequado, seja pela via enteral ou parenteral (Remillard e Tatcher, 1989).

A nutrição enteral deve ser considerada um ponto importante no plano terapêutico de animais criticamente doentes, hospitalizados ou não. A deficiência ou falta de ingestão de alimentos pode levar à fraqueza muscular, disfunção do sistema imune e demora na cicatrização de feridas. A nutrição enteral é importante para manter normais as funções enzimáticas intestinais e pancreáticas e, também, evitar a estase biliar (Crowe e Devey, 2000). A técnica utilizada neste trabalho foi a gastrostomia guiada por endoscopia. Esse método de alimentação enteral pode ser utilizado como suporte nutricional prolongado em qualquer animal que apresente anorexia ou disfagia (Abbod e Buffington., 1993; Marks, 1998).

Quando o trato gastrintestinal não apresentar condições de digerir e absorver os nutrientes, o suporte enteral é contra-indicado. Nesse caso, a via parenteral é preferível (Burrows, 1981; McConnell, 2001).

A fluidoterapia intravenosa é indicada principalmente quando o grau de desidratação é alto, necessitando-se de uma resposta rápida para salvar a vida do animal, ou quando o animal está muito deprimido ou, ainda, quando apresenta perda aguda de fluidos (DiBartola, 1992). Ela é comumente utilizada em clínicas veterinárias para a manutenção de animais anoréxicos ou impossibilitados de se alimentarem. É uma prática quase sempre utilizada de maneira aleatória, mas fundamental para a manutenção ou correção do equilíbrio hidroeletrolítico do animal. Seu uso não evita nem minimiza o balanço energético negativo, resultante da anorexia e do aumento do requerimento energético, normalmente encontrados em animais doentes.

O objetivo deste trabalho foi verificar a influência de diferentes formas de alimentação clínica sobre características clínicas e hematológicas, em cães.

\section{MATERIAL E MÉTODOS}

Utilizaram-se 20 cães, sem raça definida, com idade variando entre dois e oito anos e peso entre 11,1 a $28,6 \mathrm{~kg}$. Os animais foram alojados em canis coletivos para cumprirem o período de adaptação de 15 dias, onde receberam ração super premium ${ }^{1}$.

Os animais foram agrupados de acordo com o sexo e o peso e divididos aleatoriamente em quatro grupos experimentais (cinco animais por grupo, sendo três machos e duas fêmeas). No grupo I - controle receberam ração super premium comercial ${ }^{1}$, duas vezes ao dia, durante sete dias. A quantidade de ração diária individual foi calculada de acordo com seus requisitos energéticos, levando-se em conta o peso metabólico e a densidade energética da ração. $\mathrm{Na}$ Tab. 1 mostra-se a quantidade de ração recebida, de acordo com o peso metabólico (a água foi fornecida à vontade).

No grupo II - fluidoterapia intravenosa - os animais receberam exclusivamente solução

\footnotetext{
${ }^{1}$ Premier cães adultos - Premier pet -São Paulo
} 
ringer lactato e solução glicofisiológica $5 \%$, às 8 horas, durante sete dias. O volume calculado de fluido foi $60 \mathrm{ml} / \mathrm{kg}$ de peso corporal (DiBartola, 1992), metade fornecido pela solução de ringer lactato e metade pela solução de glicofisiológica a 5\%. O acesso venoso foi através da cateterização transdérmica da veia cefálica. $\mathrm{Na}$ Tab. 2 mostra-se o volume diário das duas soluções, administrado de acordo com o peso vivo.
No grupo III - nutrição enteral - utilizou-se o método de gastrostomia, tendo sua implantação guiada por endoscopia, como método de fornecimento do suporte enteral. A dieta utilizada foi a mesma do período de adaptação. A quantidade de ração diária individual foi calculada de acordo com os requisitos energéticos, levando-se em conta o peso metabólico e a densidade energética da ração.

Tabela 1. Quantidade diária de ração comercial ${ }^{\mathrm{a}}$, em gramas, fornecida por animal do grupo I (controle)

\begin{tabular}{lcccccc}
\hline Animal & Sexo & Peso $(\mathrm{kg})$ & Peso metabólico $^{1}$ & ${\text { REM }(\mathrm{Kcal})^{2}}^{2}$ & ${\text { EM Ração }(\mathrm{Kcal})^{3}}^{3}$ & ${\text { Ração }(\mathrm{g} / \mathrm{dia})^{4}}^{4}$ \\
\hline 1 & $\mathrm{~F}$ & 16,7 & 8,3 & 1156,4 & 4130,0 & 280,0 \\
2 & $\mathrm{M}$ & 14,2 & 7,3 & 1024,8 & 4130,0 & 248,2 \\
3 & $\mathrm{M}$ & 13,4 & 7,0 & 980,0 & 4130,0 & 237,3 \\
4 & $\mathrm{~F}$ & 18,5 & 8,9 & 1248,8 & 4130,0 & 302,4 \\
5 & $\mathrm{M}$ & 12,0 & 6,5 & 903,0 & 4130,0 & 218,6 \\
\hline
\end{tabular}

${ }^{a}$ Premier cães adultos - Premier pet -Sâo Paulo

${ }^{1}$ Peso metabólico $(\mathrm{PM})=(\text { peso vivo })^{0,75}$.

${ }^{2}$ Requisito energético para manutenção $(\mathrm{REM})=140 \times \mathrm{PM}$.

${ }^{3}$ Energia metabolizável da ração $(\mathrm{EM})=3,075+0,066$ (extrato etéreo dieta) (Case, 1998).

${ }^{4}$ Quantidade de ração fornecida (arredondada da ração calculada): animal $1=280 \mathrm{~g}$; animal $2=250 \mathrm{~g}$; animal $3=240 \mathrm{~g}$; animal $4=$ $305 \mathrm{~g}$; animal $5=220 \mathrm{~g}$.

Tabela 2. Volume diário das soluções glicofisiológicas 5\% e ringer lactado por animal do grupo II (fluidoterapia intravenosa)

\begin{tabular}{lccccccc}
\hline Animal & Sexo & $\begin{array}{c}\text { Peso } \\
(\mathrm{kg})\end{array}$ & $\begin{array}{c}\text { Volume total } \\
(\mathrm{ml})\end{array}$ & $\begin{array}{c}\text { Solução } \\
\text { glicofisiológica }(\mathrm{ml})^{1}\end{array}$ & $\begin{array}{c}\text { Ringer lactato } \\
(\mathrm{ml})^{1}\end{array}$ & $\begin{array}{c}\text { Kcal fornecida } \\
\text { por dia }\end{array}$ & $\begin{array}{c}\text { Necessidade } \\
\text { calórica/dia }\end{array}$ \\
\hline 1 & $\mathrm{~F}$ & 29,8 & 1788 & 894 & 894 & 161,1 & 1785,0 \\
2 & $\mathrm{M}$ & 15 & 900 & 450 & 450 & 80,6 & 1066,8 \\
3 & $\mathrm{~F}$ & 15,5 & 930 & 465 & 465 & 89,5 & 1093,4 \\
4 & $\mathrm{M}$ & 11,1 & 666 & 333 & 333 & 62,7 & 851,2 \\
5 & $\mathrm{M}$ & 14,8 & 888 & 444 & 444 & 80,6 & 1055,6 \\
\hline
\end{tabular}
$5=450 \mathrm{ml}$

O volume diário de água foi calculado como sendo três vezes a quantidade diária, em gramas, da ração (Lewis, 1994; Gross et al., 2000). A dieta foi dividida em três administrações diárias, às 8,14 e 18 horas, durante sete dias. Na Tab. 3 apresentam-se a quantidade de ração e o volume de água diários, administrados de acordo com o peso metabólico.

Tabela 3. Quantidade de ração e volume de água diário fornecido por animal do grupo III (nutrição enteral)

\begin{tabular}{lccccccc}
\hline Animal & Sexo & $\begin{array}{c}\text { Peso vivo } \\
(\mathrm{kg})\end{array}$ & $\begin{array}{c}\text { Peso } \\
\text { metabólico }\end{array}$ & $\begin{array}{c}\text { REM } \\
(\mathrm{kcal})\end{array}$ & $\begin{array}{c}\text { EM ração } \\
(\mathrm{kcal})\end{array}$ & $\begin{array}{c}\text { Ração } \\
(\mathrm{g}) / \mathrm{dia}^{1}\end{array}$ & $\begin{array}{c}\text { Volume de água } \\
(\mathrm{ml}) / \mathrm{dia}^{2}\end{array}$ \\
\hline 1 & $\mathrm{~F}$ & 19,1 & 9,1 & 1279,6 & 4130,0 & 309,8 & 929,5 \\
2 & $\mathrm{M}$ & 16,0 & 8,0 & 1120,0 & 4130,0 & 271,2 & 813,6 \\
3 & $\mathrm{M}$ & 13,1 & 6,9 & 964,6 & 4130,0 & 233,6 & 700,7 \\
4 & $\mathrm{~F}$ & 12,0 & 6,5 & 903,0 & 4130,0 & 218,6 & 655,9 \\
5 & $\mathrm{M}$ & 18,0 & 8,7 & 1223,6 & 4130,0 & 296,3 & 888,8 \\
\hline
\end{tabular}

${ }^{1}$ Ração fornecida arredondada da ração calculada; animal 1=310g; animal 2=270g; animal 3=235g; animal $4=220 \mathrm{~g}$; animal $5=$ $300 \mathrm{~g}$.

${ }^{2}$ Volume diário de água = três vezes a quantidade de ração $(\mathrm{g})$ fornecida. Volume arredondado; animal $1=930 \mathrm{ml}$; animal $2=815 \mathrm{ml}$; animal $3=705 \mathrm{ml}$; animal $4=660 \mathrm{ml}$; animal $5=900 \mathrm{ml}$.

$\mathrm{REM}=$ requisito energético de manutenção, $\mathrm{EM}=$ energia metabolizável. 
No grupo IV - nutrição parenteral - os animais receberam o suporte nutricional total durante sete dias, sendo o tratamento iniciado às 8horas. O acesso venoso foi através da veia cefálica. Para a obtenção da solução parenteral total, foram utilizadas soluções de aminoácidos, soluções de lipídio e de glicose 50\%. Para o cálculo do volume participante de cada solução na solução parenteral final, foram utilizadas as recomendações de Armstrong e Lippert (1988), que consideram apenas as calorias fornecidas pelas soluções de glicose e lipídio. O volume das soluções administradas a cada animal é apresentado na Tab. 4. Após receberem soluções parenterais, os animais foram submetidos à fluidoterapia intravenosa, pela administração das soluções de ringer lactato e fisiológica a $0,9 \%$, utilizando-se os mesmos cálculos do grupo II, com o objetivo de se evitar desidratação, visto que as soluções parenterais eram hipertônicas.

Tabela 4. Volume diário fornecido das soluções de aminoácidos (AA), lipídios e glicose por animal do grupo IV (nutrição parenteral)

\begin{tabular}{lcccccccc}
\hline Animal & Sexo & $\begin{array}{c}\text { Peso } \\
(\mathrm{kg})\end{array}$ & $\begin{array}{c}\text { Peso } \\
\text { metabólico }\end{array}$ & $\begin{array}{c}\text { REM } \\
(\mathrm{kcal})\end{array}$ & $\begin{array}{c}\text { Necessidade } \\
\text { protéica }^{1}(\mathrm{~g})\end{array}$ & $\begin{array}{c}\text { Solução AA } \\
(\mathrm{ml})\end{array}$ & $\begin{array}{c}\text { Solução } \\
\text { lipídica }^{2}(\mathrm{ml})\end{array}$ & $\begin{array}{c}\text { Solução } \\
\text { glicose }^{2}(\mathrm{ml})\end{array}$ \\
\hline 1 & $\mathrm{M}$ & 14,4 & 7,0 & 1034,6 & 57,6 & 576,0 & 258,7 & 304,3 \\
2 & $\mathrm{~F}$ & 16,7 & 8,3 & 1156,4 & 66,8 & 668,0 & 289,1 & 340,1 \\
3 & $\mathrm{M}$ & 11,5 & 6,2 & 873,6 & 46,0 & 460,0 & 218,4 & 256,9 \\
4 & $\mathrm{M}$ & 18,1 & 8,8 & 1229,2 & 72,4 & 724,0 & 307,3 & 361,5 \\
5 & $\mathrm{~F}$ & 22,0 & 10,2 & 1422,4 & 88,0 & 880,0 & 355,6 & 418,4 \\
\hline
\end{tabular}

${ }^{1}$ Necessidades protéicas $=4 \mathrm{~g}$ de proteína $/ \mathrm{kg}$ de peso vivo.

${ }^{2}$ Solução de aminoácidos (Aminosteril 10\%) = 10g proteína/100ml; solução de lipídios (Lipovenus 20\%) = 2000Kcal/1000ml; solução de glicose $50 \%=1700 \mathrm{Kcal} / 1000 \mathrm{ml}$.

Antes de instituir os tratamentos, todos os animais permaneceram em jejum por 48 horas. Esse procedimento deveu-se ao fato de os animais serem submetidos ao exame endoscópico do trato gastrintestinal. Somente os do grupo III necessitavam ser submetidos à endoscopia para a colocação da sonda gástrica, mas o procedimento foi o mesmo para todos os animais.

Eles foram avaliados clinicamente quanto ao peso vivo (PV), temperatura retal (TR), comportamento em resposta a estímulo externo e escore fecal. Os pesos foram obtidos antes do jejum, no primeiro, no quarto e no último dia de tratamento, sempre nos mesmos horários, às 13 horas. Foram realizadas duas mensurações diárias para a temperatura retal, sendo a primeira entre 7 e 8 horas e a segunda entre 20 e 21 horas. O comportamento dos animais foi observado diariamente entre 7 e 8 horas e também durante a colheita de material, observando-se sua reação à manipulação e às punções.

O escore fecal, avaliado diariamente, foi classificado em fezes consistentes, pastosas e líquidas, logo após a defecação. Observou-se, ainda, a ocorrência de flebite nas veias cefálicas, visto que a administração das soluções nos animais dos grupos II e IV ocorreu por esse acesso.

Colheram-se amostras de sangue por venopunção central da jugular externa, em tubos a vácuo, com anticoagulante (EDTA). A primeira, antes do início do experimento, e as outras, diariamente, sempre entre 7 e 8 horas. $\mathrm{O}$ hemograma constitui-se de eritrograma e hemograma.

O delineamento experimental foi inteiramente ao acaso, em parcelas subdivididas, tendo os grupos na parcela, e os dias e coletas e suas interações na subparcela. Para as variáveis com valores numéricos, como hemograma, bioquímica, peso e gasometria, utilizou-se o teste de Student Newman Keuls para a comparação das médias.

\section{RESULTADOS E DISCUSSÃO}

Não houve diferenças entre os grupos nem dentro de grupo $(\mathrm{P}>0,05)$ quanto aos pesos obtidos antes do jejum, depois do jejum (primeiro dia tratamento), no quarto e no sétimo dia de tratamento (Tab. 5). 
Aspectos clínicos e hematológicos...

Tabela 5. Peso médio $(\mathrm{kg})$ de cães submetidos a diferentes tratamentos nutricionais (grupo I - controle, ração comercial; grupo II - fluidoterapia intravenosa; grupo III - nutrição enteral; grupo IV - nutrição parenteral)

\begin{tabular}{ccccc} 
Dia $^{1}$ & Grupo 1 & Grupo II & Grupo III & Grupo IV \\
\hline 0 & $15,4 \pm 2,5$ & $17,5+7,2$ & $16,5+3,1$ & $17,12+3,9$ \\
1 & $15,0+2,6$ & $16,7 \pm 6,9$ & $15,7 \pm 3,1$ & $16,5 \pm 3,9$ \\
4 & $14,9 \pm 2,4$ & $15,9 \pm 7,2$ & $16,1 \pm 3,0$ & $16,6 \pm 4,0$ \\
7 & $14,9 \pm 2,6$ & $15,3 \pm 7,4$ & $15,7 \pm 2,9$ & $16,3 \pm 3,8$ \\
\hline
\end{tabular}

Dia 1 = peso antes do jejum; dia 2 = peso após o jejum (dia 1 do tratamento); dia $3=$ peso no quarto dia de tratamento; dia $4=$ peso no último dia de tratamento (sétimo dia).(P>0,05).

Quanto à perda de peso, observou-se diferença $(\mathrm{P}<0,05)$ entre o grupo da fluidoterapia (maior perda) e os demais (Tab. 6). Esta observação é semelhante à de Gross et al. (2000) e se justifica pelo fato de os animais desse grupo ficarem em jejum alimentar completo durante nove dias (dois dias do período de jejum e sete dias do período de tratamento). A perda de peso não foi percebida somente pela aferição do peso vivo. Clinicamente os animais do grupo apresentaramse, ao final do sétimo dia de tratamento, com estado corporal ruim, isto é, as protuberâncias ósseas, como íleo, ísquio, costado e processos espinhosos das vértebras torácicas e lombares, estavam proeminentes. Os animais desse grupo receberam quantidades de energia abaixo de suas necessidades metabólicas (Tab. 2), portanto, entraram em estado de catabolismo de gorduras e proteínas, justificando a perda de peso ocorrida. Redução prolongada no consumo de alimentos basicamente leva à deficiência energética crônica. Como resultado, há perda de peso corporal enquanto as reservas energéticas corporais são utilizadas (gorduras e proteína muscular) (Gross et al., 2000). Assim, apesar da manutenção hidroeletrolítica fornecida pela fluidoterapia, esse método não forneceu suporte nutricional adequado para a manutenção do escore e/ou peso corporal. Essa condição foi traduzida por perda de peso de $14,0 \pm 5,7 \%$ do peso vivo em nove dias.

Houve diferença (Tab. 7) entre a primeira mensuração de temperatura no grupo I e a primeira medida do grupo III e do grupo IV, no dia três $(\mathrm{P}<0,05)$. Ela foi maior no grupo I (acima do valor de referência). Os outros valores permaneceram dentro da faixa de normalidade para a temperatura retal. Não houve aumento no número total de leucócitos (Tab. 8) para os animais do grupo I, portanto, não há como explicar o aumento de temperatura retal ocorrida no dia três. De acordo com Kleiber (1961) e Aiello (2001), há aumento na produção de calor quando ocorre aumento do catabolismo lipídico e protéico, levando a um estado de hipertermia.

Tabela 6. Perda de peso e percentual de perda de peso de cães submetidos a diferentes tratamentos nutricionais (grupo I - controle, ração comercial; grupo II -fluidoterapia intravenosa; grupo III nutrição enteral; grupo IV - nutrição parenteral)

\begin{tabular}{lcc}
\hline \multirow{2}{*}{ Grupos } & \multicolumn{2}{c}{ Perda de peso } \\
\cline { 2 - 3 } & $\mathrm{kg}$ & $\%$ \\
\hline $\mathrm{I}$ & $0,5 \mathrm{~B} \pm 0,3$ & $3,5 \mathrm{~B} \pm 1,9$ \\
II & $2,2 \mathrm{~A} \pm 0,5$ & $14,0 \mathrm{~A} \pm 5,7$ \\
III & $0,8 \mathrm{~B} \pm 0,4$ & $4,6 \mathrm{~B} \pm 2,2$ \\
IV & $0,8 \mathrm{~B} \pm 0,6$ & $5,0 \mathrm{~B} \pm 3,8$ \\
\hline
\end{tabular}

Médias seguidas por letras distintas na mesma coluna diferem entre si $(\mathrm{P}<0,05)$.

O comportamento dos animais foi classificado em normal, apático e extremamente apático. Três animais do grupo II tornaram-se apáticos (responderam a estímulos mais lentamente que os animais do grupo-controle) a partir do quarto dia de tratamento até o final. Segundo Gross et al. (2000), animais em deprivação alimentar prolongada reagem em menor intensidade a estímulos externos, provavelmente pela diminuição da taxa metabólica basal. O comportamento dos animais dos demais grupos foi normal. 
Tabela 7. Médias diárias da temperatura retal $\left({ }^{\circ} \mathrm{C}\right)$ de cães submetidos a diferentes tratamentos nutricionais (grupo I - controle, ração comercial; grupo II - fluidoterapia intravenosa; grupo III - nutrição enteral; grupo IV - nutrição parenteral)

\begin{tabular}{lcccccccc}
\hline \multirow{2}{*}{ Dia } & \multicolumn{2}{c}{ Grupo I } & \multicolumn{2}{c}{ Grupo II } & \multicolumn{2}{c}{ Grupo III } & \multicolumn{2}{c}{ Grupo IV } \\
\cline { 2 - 8 } & Coleta 1 & Coleta 2 & Coleta 1 & Coleta 2 & Coleta 1 & Coleta 2 & Coleta 1 & Coleta 2 \\
\hline 1 & $38,9 \mathrm{AB} \pm 0,1$ & $38,6 \mathrm{~A} \pm 0,1$ & $39,0 \pm 0,2$ & $38,4 \mathrm{~B} \pm 0,4$ & $39,4 \mathrm{~A} \pm 0,8$ & $38,7 \pm 0,4$ & $39,0 \pm 0,2$ & $38,5 \pm 0,8$ \\
2 & $38,6 \mathrm{~B} \pm 0,3$ & $38,9 \mathrm{~A} \pm-$ & $38,7 \pm 0,3$ & $38,8 \mathrm{AB} \pm 0,1$ & $39,3 \mathrm{AB} \pm 0,5$ & $39,3 \pm 0,7$ & $38,5 \pm 0,2$ & $39,0 \pm 0,7$ \\
3 & $40,5 \mathrm{Aa} \pm-$ & $38,7 \mathrm{Aab} \pm 0,7$ & $38,8 \mathrm{ab} \pm 0,2$ & $39,3 \mathrm{ABab} \pm 0,5$ & $38,5 \mathrm{ABb} \pm 0,5$ & $38,9 \mathrm{ab} \pm 0,5$ & $38,5 \mathrm{~b} \pm 0,2$ & $38,9 \mathrm{ab} \pm 0,2$ \\
4 & $38,7 \mathrm{ABab} \pm 0,5$ & $39,0 \mathrm{Aab} \pm 0,6$ & $38,9 \mathrm{ab} \pm 0,5$ & $39,6 \mathrm{Aa} \pm 0,9$ & $38,6 \mathrm{ABb} \pm 0,5$ & $39,2 \mathrm{ab} \pm 0,3$ & $38,6 \mathrm{ab} \pm 0,4$ & $39,1 \mathrm{ab} \pm 0,4$ \\
5 & $39,2 \mathrm{ABa} \pm 0,4$ & $36,9 \mathrm{Bb} \pm 4,6$ & $38,7 \mathrm{a} \pm 0,8$ & $39,5 \mathrm{ABa} \pm 0,8$ & $39,0 \mathrm{ABa} \pm 0,5$ & $38,9 \mathrm{a} \pm 0,3$ & $38,5 \mathrm{a} \pm 0,5$ & $38,9 \mathrm{a} \pm 0,5$ \\
6 & $38,7 \mathrm{AB} \pm 0,2$ & $38,9 \mathrm{~A} \pm 0,9$ & $38,8 \pm 0,7$ & $39,0 \mathrm{AB} \pm 0,5$ & $38,2 \mathrm{~B} \pm 0,8$ & $38,4 \pm 0,6$ & $39,0 \pm 0,4$ & $39,1 \pm 0,7$ \\
7 & $38,7 \mathrm{AB} \pm 0,3$ & $39,0 \mathrm{~A} \pm-$ & $38,8 \pm 0,8$ & $38,6 \mathrm{AB}+1,3$ & $38,8 \mathrm{AB} \pm 0,6$ & $39,0 \pm 0,2$ & $38,7 \pm 0,2$ & $39,3 \pm 0,8$ \\
Total & $38,68 \pm 1,44$ & $38,9 \pm 0,7$ & $38,9 \pm 0,6$ & $38,8 \pm 0,5$ & \\
\hline
\end{tabular}

Médias seguidas por letras maiúsculas distintas na mesma coluna ou por letras minúsculas distintas na mesma linha, diferem entre si $(\mathrm{P}<0,05)$.

Tabela 8. Leucócitos totais $\left(\mathrm{x} 10^{3} / \mathrm{mm}^{3}\right.$ ) de cães submetidos a diferentes tratamentos nutricionais (grupo I - controle, ração comercial; grupo II - fluidoterapia intravenosa; grupo III - nutrição enteral; grupo IV nutrição parenteral)

\begin{tabular}{lcccc}
\hline Dia & Grupo I & Grupo II & Grupo III & Grupo IV \\
\hline 0 & $12,6 \pm 3,7$ & $11,5 \pm 6,1$ & $17,6 \mathrm{BC} \pm 7,4$ & $13,6 \pm 5,9$ \\
1 & $13,1 \mathrm{ab} \pm 5,8$ & $12,4 \mathrm{ab} \pm 4,7$ & $19,9 \mathrm{ABCa} \pm 10,3$ & $11,3 \mathrm{~b} \pm 6,1$ \\
2 & $12,6 \mathrm{~b} \pm 4,6$ & $15,2 \mathrm{~b} \pm 8,2$ & $25,5 \mathrm{Aa} \pm 8,9$ & $13,3 \mathrm{~b} \pm 7,3$ \\
3 & $13,3 \mathrm{~b} \pm 7,2$ & $13,4 \mathrm{~b} \pm 7,6$ & $21,7 \mathrm{ABb} \pm 10,1$ & $14,0 \mathrm{~b} \pm 5,0$ \\
4 & $12,8 \pm 5,6$ & $16,7 \pm 8,8$ & $19,3 \mathrm{ABC} \pm 6,7$ & $15,8 \pm 7,1$ \\
5 & $12,4 \pm 5,4$ & $15,6 \pm 7,0$ & $15,9 \mathrm{BC} \pm 6,0$ & $16,0 \pm 6,8$ \\
6 & $14,1 \pm 8,8$ & $12,9 \pm 3,9$ & $14,1 \mathrm{C} \pm 4,4$ & $16,4 \pm 3,5$ \\
7 & $15,1 \pm 7,7$ & $11,1 \pm 6,1$ & $13,2 \mathrm{C} \pm 4,0$ & $13,6 \pm 4,4$ \\
\hline
\end{tabular}

Médias seguidas por letras maiúsculas distintas na mesma coluna ou por letras minúsculas distintas na mesma linha, diferem entre si $(\mathrm{P}<0,05)$.

Valores de referência: 6,00 a 17,00 x $10^{3}$ (Jain, 1986).

Todos os animais do grupo IV apresentaram flebite na veia cefálica, no terceiro dia de tratamento, quando, então, realizou-se a troca do catéter. Essa alteração clínica foi manifestada, primeiramente, por diminuição no fluxo de gotejamento dos equipos. Apesar da flebite, não ocorreu variação no número total de leucócitos (Tab. 8). A velocidade de gotejamento das soluções parenterais foi elevada, o que pode explicar a ocorrência das flebites, uma vez que as soluções apresentavam alta osmolaridade. Segundo Armstrong e Lippert (1988), a velocidade de administração deve ser de 62 $\mathrm{ml} /$ hora para cães e de $19 \mathrm{ml} /$ hora para gatos. Para a administração da nutrição parenteral total (NPT), recomenda-se a venopunção central. Para tanto, deve-se posicionar percutaneamente na veia jugular dos animais um catéter de poliuretano ou silicone (Remillard e Tatcher, 1989; Jackson e Vail, 1993; Lewis, 1994). Entretanto, devido ao custo da implantação do catéter na jugular e à dificuldade de sua manutenção, utilizaram-se vasos periféricos, como a veia cefálica. O volume das soluções parenterais foi grande por não ter sido considerado o cálculo da energia fornecida pelas proteínas quando se realizou o preparo das soluções. Em cães, a proteína adicionada à NPT não é considerada no cálculo calórico, pois contribui com apenas pequena quantidade do cálculo total (Armstrong e Lippert, 1988). Entretanto, Ravasco e Camilo (2003) afirmaram que o fornecimento de calorias protéicas, não calculadas no requisito energético para cães, deve ser considerado, visto que pode levar a um quadro de hipernutrição. Então, após inspeção do catéter e palpação da veia cefálica, notou-se que ela estava extremamente rígida e que a sua manipulação causava dor. Dois animais do grupo IV, além das alterações acima, apresentaram ulceração da pele no local onde o catéter foi introduzido. Essas feridas foram tratadas com soluções de iodo povidona. No sexto dia de tratamento, os animais apresentaram novamente as alterações descritas, mas sem ulcerações de pele. Realizou-se, mais uma vez, a troca do catéter, para a cefálica que fora acessada inicialmente, exceto nos dois animais citados, 
devido à ferida na pele. Neles recorreu-se ao acesso venoso mediante a venopunção da veia tarsal. Realizou-se a troca dos catéteres dos animais do grupo II no terceiro e sexto dias de tratamento. Não houve sinais clínicos de flebite nesses animais, mas em dois deles ocorreram feridas na pele, próximas à inserção do tubo extensor e ao catéter, provavelmente devido ao excesso de compressão exercida pela bandagem ou à reação alérgica ao esparadrapo.

Em relação ao escore fecal, um animal do grupo I apresentou fezes pastosas, amareloamarronzadas, no segundo dia de tratamento, que se normalizaram no terceiro dia e assim permaneceram até o final do tratamento. Nesse mesmo animal, no mesmo dia, ocorreu episódio de vômito. Em um animal do grupo IV, observaram-se fezes pastosas, em pequena quantidade e escuras, no quarto dia de tratamento, e em outro, isso ocorreu no quinto dia de tratamento. Os outros animais desse grupo não apresentaram fezes durante o período de tratamento. Em um animal do grupo II, verificaram-se fezes líquidas, escuras, em pequena quantidade, no quarto dia de tratamento. Como estava em jejum, quanto à alimentação via oral, o trato gastrintestinal não estava recebendo alimentos e, portanto, deveria estar com motilidade propulsiva bastante diminuída. Entretanto, algum alimento apreendido antes do período de jejum poderia ainda estar no trato gastrintestinal e ser eliminado nas fezes nos dias mencionados acima. Os outros animais do grupo II não apresentaram fezes. Em dois animais do grupo III, observaram-se fezes líquidas, amarelo-amarronzadas, no segundo dia de tratamento, provavelmente devido à não adaptação, ainda, do método de tratamento.

Nos animais do grupo II, observou-se anemia normocítica normocrômica, mas os valores de hemácias, hemoglobina, volume corpuscular médio e concentração de hemoglobina corpuscular média não diferiram entre os tratamentos $(\mathrm{P}>0,05)$. Na Tab. 9 mostram-se os valores de hemácias para os diferentes grupos.

Tabela 9. Valores de hemácias $\left(\times 10^{6} / \mathrm{mm}^{3}\right)$ de cães submetidos a diferentes tratamentos nutricionais (grupo I - controle, ração comercial; grupo II - fluidoterapia intravenosa; grupo III - nutrição enteral; grupo IV - nutrição parenteral)

\begin{tabular}{lllll}
\hline DIA & Grupo I & Grupo II & Grupo III & Grupo IV \\
\hline 0 & $6,1 \pm 0,7$ & $5,8 \pm 1,0$ & $6,0 \pm 0,6$ & $6,1 \pm 1,1$ \\
1 & $6,2 \pm 1,1$ & $5,4 \pm 0,9$ & $6,0 \pm 0,7$ & $6,2 \pm 0,9$ \\
2 & $6,0 \pm 1,1$ & $5,2 \pm 1,1$ & $5,8 \pm 0,5$ & $5,7 \pm 0,7$ \\
3 & $5,8 \pm 1,0$ & $5,1 \pm 1,1$ & $5,5 \pm 0,7$ & $6,2 \pm 1,2$ \\
4 & $5,8 \pm 0,9$ & $5,1 \pm 1,0$ & $5,3 \pm 0,5$ & $5,7 \pm 1,1$ \\
5 & $5,7 \pm 0,8$ & $5,0 \pm 0,8$ & $5,4 \pm 0,6$ & $5,7 \pm 1,0$ \\
6 & $5,6 \pm 1,0$ & $4,9 \pm 0,7$ & $5,1 \pm 0,4$ & $5,5 \pm 0,8$ \\
7 & $5,9 \pm 0,8$ & $5,0 \pm 0,8$ & $5,5 \pm 0,8$ & $5,4 \pm 0,7$ \\
\hline Total & $5,9 \pm 0,9$ & $5,2 \pm 0,9$ & $5,6 \pm 0,6$ & $5,8 \pm 0,9$ \\
\hline$(\mathrm{P}>0,05)$. Valores de referência: $5,50 \mathrm{a} 8,50 \times 10^{6} / \mathrm{mm}^{3}$ (Jain, 1986$)$. & &
\end{tabular}

Nos animais do grupo IV, o número de leucócitos totais, nos dias um, dois e três, foi maior do que nos dos grupos II e IV $(\mathrm{P}<0,05)$. Para colocação da sonda gástrica, houve ocorrência de lesão tecidual inerente à própria técnica. Lesões teciduais levam à liberação de mediadores inflamatórios, que, por sua vez, determinam aumento dos leucócitos totais circulantes (Jain, 1986). Esse aumento dos leucócitos totais deveu-se principalmente ao aumento absoluto de neutrófilos segmentados $(\mathrm{P}<0,05)$ em relação aos animais do grupo I, ocorrido no dia 1 (grupo $\mathrm{I}=8,1 \pm 3,2 \times 10^{3} / \mathrm{mm}^{3}$; grupo III $\left.=15,9 \pm 9,5 \times 10^{3} / \mathrm{mm}^{3}\right)$, no dia 2 (grupo I $=7,4 \pm 2,8 \times 10^{3} / \mathrm{mm}^{3}$; grupo III = $19,5 \pm 7,5 \times 10^{3} / \mathrm{mm}^{3}$ ), no dia 3 (grupo $\mathrm{I}=$ $8,1 \pm 5,5 \times 10^{3} / \mathrm{mm}^{3}$; grupo III = $16,2 \pm 8,7 \times 10^{3} / \mathrm{mm}^{3}$ ) e no dia 4 (grupo $\mathrm{I}=$ $8,1 \pm 3,5 \times 10^{3} / \mathrm{mm}^{3}$; grupo III = $14,4 \pm 5,3 \times 10^{3} / \mathrm{mm}^{3}$ ). No dia 1 , houve aumento nos valores absolutos de neutrófilos bastonetes nos animais do grupo III $\left(0,68 \pm 0,8 \times 10^{3} / \mathrm{mm}^{3}\right)$, quando comparados aos dos demais grupos (grupo I $=0,0 \pm 0,0 \times 10^{3} / \mathrm{mm}^{3}$; grupo II $=0,12 \pm$ $0,19 \times 10^{3} / \mathrm{mm}^{3}$; grupo IV $=0,23 \pm 0,36 \times$ $10^{3} / \mathrm{mm}^{3}$ ). 


\section{CONCLUSÕES}

A manutenção de animais anoréticos ou impossibilitados de se alimentarem não dever ser realizada apenas com o uso da fluidoterapia. Um suporte nutricional parenteral ou enteral se faz necessário para a manutenção de parâmetros clínicos e laboratoriais, dentro dos valores de referência para cães. A gastrostomia mostrou ser um método eficiente, devendo ser empregada como alternativa para a alimentação enteral em cães. Deve-se considerar o valor da energia fornecida pelas proteínas no cálculo da nutrição parenteral total, visando diminuir o volume infundido. Velocidade de infusão elevada, por acesso venoso periférico, aumenta a ocorrência de flebites.

\section{REFERÊNCIAS BIBLIOGRÁFICAS}

ABBOD, S.K.; BUBBIGTON, C.A. Nutritional support of hospitalized patients. In: SLATTER, D. Textbook of small animal surgery. 2.ed. Philadelphia: W.B. Saunders, 1993. cap.5, p.6383.

AIELLO, S.E. (Ed.). Manual Merck de veterinária. 8.ed. São Paulo: Roca, 2001. p.927951.

ARMSTRONG, P.J.; LIPPERT, A.C. Select aspects of enteral and parenteral nutrition support. Sem. Vet. Med. Surg. (Small Anim.), v.3, p.216-226, 1988.

BURROWS, C.F. Veterinary intensive care. $J$. Small Anim. Pract., v.22, p.231-235, 1981.

CASE, L.P. Nutrição canina e felina (Manual para profissionais). Madrid: Harcourt Brace, 1998. 424p.

CROWE, D.T.; DEVEY, J. Feeding tubes essential for enteral nutrition of critically-ill patients. Vet. Med., v.31, p.15-19, 2000.

DiBARTOLA, S.P. Introduction to fluid therapy. In: DiBARTOLA, S.P. Fluid therapy in small animal practice. Philadelphia: W.B. Saunders, 1992. p.321-340.
DONOGHUE, S. Nutrition support of hospitalized animals. J. Small Anim. Pract., v.33, p.183-190, 1992.

GROSS, K.L.; WEDEKIND, K.J.; COWEL, C.S. et al. Nutrients. In: HAND, M.S.; THATCHER, C.D.; REMILLARD, R.L. et al. (Eds.). Small Animal. Clinic Nutrition. 4.ed. Topeka: Mark Morris Institute, 2000. p.21-101.

JACKSON, M.W.; VAIL, D.M. Nutritional management of cats whith infection disease. Vet. Clin. North Am.: Small Anim. Pract., v.23, p.155-171, 1993.

JAIN, N.C. Schalm's veterinary hematology. 4.ed. Philadelphia: Lea \& Febiger, 1986.

KLEIBER, M. Animal temperature regulation. In: The fire of life: an introduction to animal energetics. Davis, CA: John Wiley e Sons, 1961. p.146-174.

LEWIS, L.D. Anorexia, inanition and critical care nutrition. In: Small Animal Critical Care. 3.ed. Topeka: Mark Morris Institute, 1994. cap.5, p.35-43.

MARKS, S.L. The principles and pratical application of enteral nutrition. Vet. Clinic. North Amer.: Small Anim. Pract., v.28, p.677-708, 1998.

McCONNELL, E.A. Administering total parenteral nutrition. Nursing, v.31, p.17, 2001.

RAVASCO, P.; CAMILO, M.E. The impact of fluid therapy on nutrient delivery: a prospective evaluation of practice in respiratory intensive care. Clinic. Nutrit., v.22, p.87-92, 2003.

REMILLARD, R.L.; TATCHER, C.D. Parenteral nutrition support in the mall animal patient. Vet. Clinic. North Am.: Small Anim. Pract., v.19, p.1287-1306, 1989.

SIMPSON, K.W.; ELWOOD, C.M. Techiniques for enteral nutritional support. In: WILLS, J.M.; SIMPSON, K.W. (Ed.). The Waltham book of clinical nutrition of the dog and cat. Great Britain: Pergamon, 1994. p.63-74. 